\title{
Management of C Shaped Maxillary Lateral Incisor- A Case Report
}

\author{
Dr.Shristhi Sharma,Dr.AnantRaghav Sharma \\ (MDS Endodontics, Senior Lecturer, Dept. Of Conservative Dentistry And Endodontics, Pacific Dental College \\ And Research Centre,Bhillokabedla,Udaipur, India) \\ (MDS Periodontology, Reader, Dept. Of Periodontology, Pacific Dental College,Debari,Udaipur,India)
}

\begin{abstract}
Knowledge of root canal anatomy with possible variations is of prime concern responsible for successful endodontic therapy. Cooke and Cox were the first to document the C-Shaped canal in endodontic literature. The variation exhibits a fin or web connecting the individual root canals to form an orifice similar to letter ' $C$ '. The irregular areas in C-shaped root canal system often may harbor soft tissue remnant or infected bacterial biofilm, leading to inadequate cleaning and root canal obturation.The use of the ultrasonics, (MTA Fillapex), thermoplasticizedobturation techniques, SAF system, CBCT and Endodontic Microscopes can be successfully used to treat such cases. MTA based sealers help in proper sealing of the canal and also helps in regenerating bone and inducing cementogenesis.
\end{abstract}

Keyword: C shaped canals, Fan's classification, invagination, MTA Fillapex, Ultrasonic irrigation.

\section{Introduction}

Knowledge of root canal anatomy with possible variations is of prime concern responsible for successful endodontic therapy. C-shaped canal configuration exhibited by maxillary lateral incisors and mandibular second molars is of particular interest. It is often difficult to predict and negotiate a C-shaped root canal.

According to Manning, the failure of the Hertwig's epithelial root sheath to fuse was responsible for a $\mathrm{C}$-shaped root. Such roots always exhibited the presence of a C-shaped root canal.

Cooke and Cox were the first to document the C-Shaped canal in endodontic literature. The variation exhibits a fin or web connecting the individual root canals to form an orifice similar to letter ' $\mathrm{C}$ '. It appears as a fusion between the distal and mesial roots, present either buccally or lingually. An intra-radicular ribbon shaped connection is present making this fusion irregular. This canal configurations provide a challenge for effective mechanical shaping, chemical cleansing and obtaining an impervious fluid tight seal of the entire root canal system. It is not only the configuration proving a challenge but also the unclear knowledge whether the CShaped orifice at the pulp chamber actually continues till the apex.

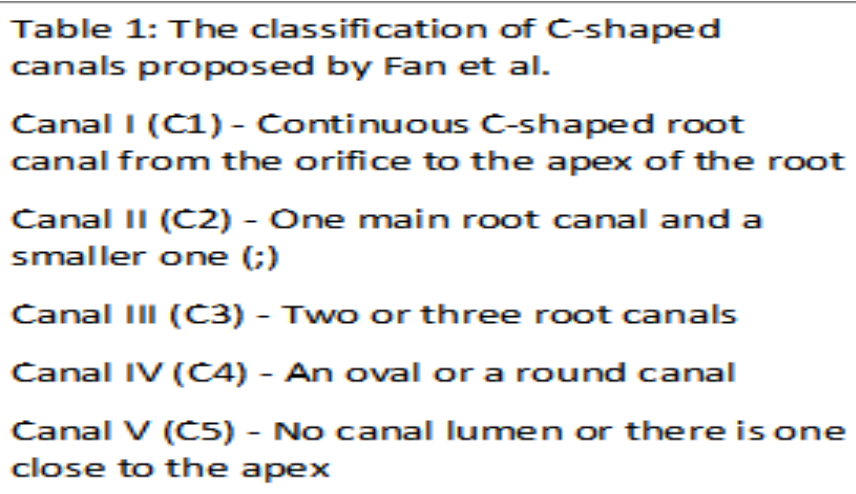

Melton in 1991 proposed the following classification of C-shaped canals based on their cross sectional shape:-

Category I: Continuous C-shaped canal running from the pulp chamber to the apex defines a C-shaped outline without any separation.

Category II: the semicolon-shaped-orifice in which dentine separates a main C-shaped canal from one mesial distinct canal.

Category III: it refers to those with two more discrete and separate canals.

Subdivision I- C-shaped orifice in the coronal third that divides into two or more discrete and separate canals that join apically. 
Subdivision II- C-shaped orifice in the coronal third that divides into two or more discrete and separate canals in the midroot to the apex.

Subdivision III- C-shaped orifice that divides into two or more discrete and separate canals in the coronal third to the apex.

In this classification there has been no clear description of the difference between categories II and III as well as the clinical significance.

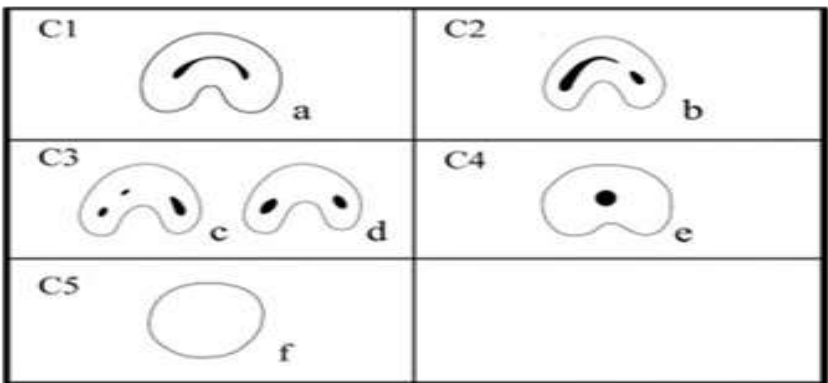

Fan’s Classification (Radiographic Classification)

Type 1- Conical or square root with a vague, radiolucent longitudinal line separating the root into distal and mesial parts. There was a mesial and distal canal that merged into one before exiting at the apical foramen (a)

Type 2- Conical or square root with a vague, radiolucent longitudinal line separating the root into distal and mesial parts. There was a mesial and distal canal and the two canals appeared to continue on their own pathway to the apex (b)

Type 3- Conical or square root with a vague, radiolucent longitudinal line separating the root into distal and mesial parts. There was a mesial and distal canal one canal curved to and superimposed on this radiolucent line when running towards the apex and other canal appeared to continue on its own pathway to the apex (c) Presence of these developmental abnormalities can alter the endodontic treatment and its prognosis. Therefore, it is necessary to diagnose their presence and evaluate the treatmentintensity before the start. This manuscript reports the endodontic treatment of a maxillary left lateral incisor with periapical lesion that had a C-shaped anatomy.

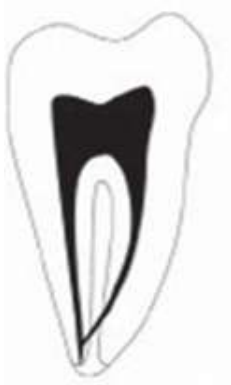

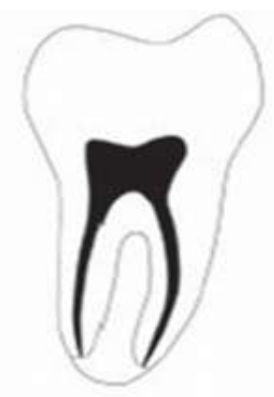

b

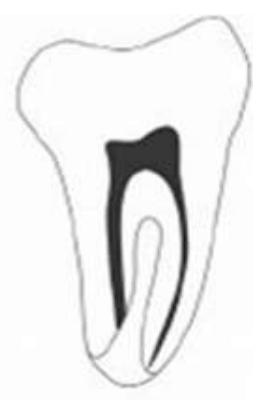

II. Case Report

A 30-year-old male patient was referred to dept. of conservative dentistry and endodontics, for pain in maxillary left lateral incisor. The patient had a non-contributory medical history. At the time of the first visit, the patient was asymptomatic. On clinical examination, the tooth was not responsive to the electronic pulp test (Parkell Electronic Division, USA) and cold test refrigerant 1, 1, 1, 2-tetrafluoro-ethane (Hygenic Endo-Ice, ColteneWhaledent, Ohio).Patient reported discomfort on percussion as well as pain on palpation of periapical area.The radiographic examination revealed an aberrant root canal anatomy and a periapical radiolucency in relation to tooth 22 (Figure 1).According to clinical and radiographic examination, the diagnosis was necrotic pulp with symptomatic apical periodontitis in relation to 22.The tooth was anesthetized and isolated with rubber dam, root canal opening of tooth 22 was done with help of Endo access bur.The Ribbon-shaped orifice of Cshaped canal started at the palatal surface of the tooth, and then swept around the distal to end at the distobuccal angle. Canal was negotiated with \#10 file (DentsplyMaillefer, Switzerland). The central canal was wide, conical and straight, while the extention of $\mathrm{C}$ shaped canal was having fins and webs. After determination of working length (Figure 2), the canal was instrumented using the step back technique and irrigated with $5.25 \%$ sodium 
hypochlorite $(\mathrm{NaOCl})$ during the instrumentation.Passive ultrasonic irrigation was performed after apical preparation till 40 no. K file using cavitron ultrasonic unit. Ultrasonic irrigation helps to debride and disinfect the canals by acoustic streaming and cavitation.After biomechanical preparation of root canal, calcium hydroxide powder mixed with saline was applied and the access cavity was temporarily sealed with Coltosol (Coltene). As the conventional techniques of cleaning and shaping cannot debride the webbed area of the $\mathrm{C}$ shaped canal.

In the next appointment after 7 days, the patient had no pain and swelling. After rubber dam isolation, calcium hydroxide was removed with master apical file and CHX irrigation. The canal was filled by MTA fillapex as a sealer along with $2 \%$ guttapercha cones using a lateral condensation technique. One week after completion of endodontic treatment, patient had no clinical symptom and the tooth was restored with composite resin (Brilliant, Dentsply). At 12 month-follow up visit, the patient reported no symptoms and the radiograph showed healing of periapical lesion.

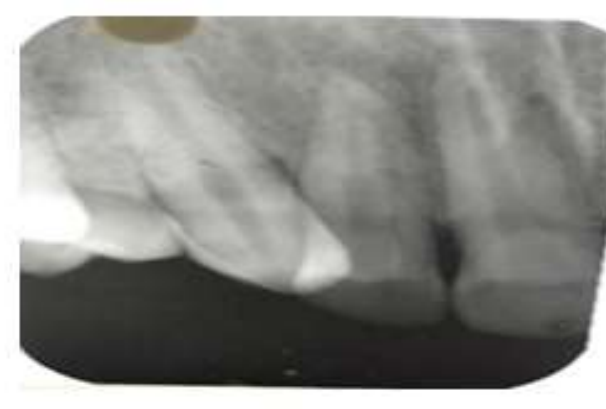

Figure 1:- Preoperative IOPA

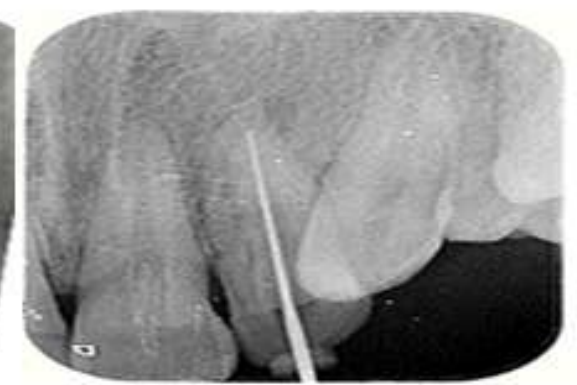

Figure 2:- Working Length IOPA
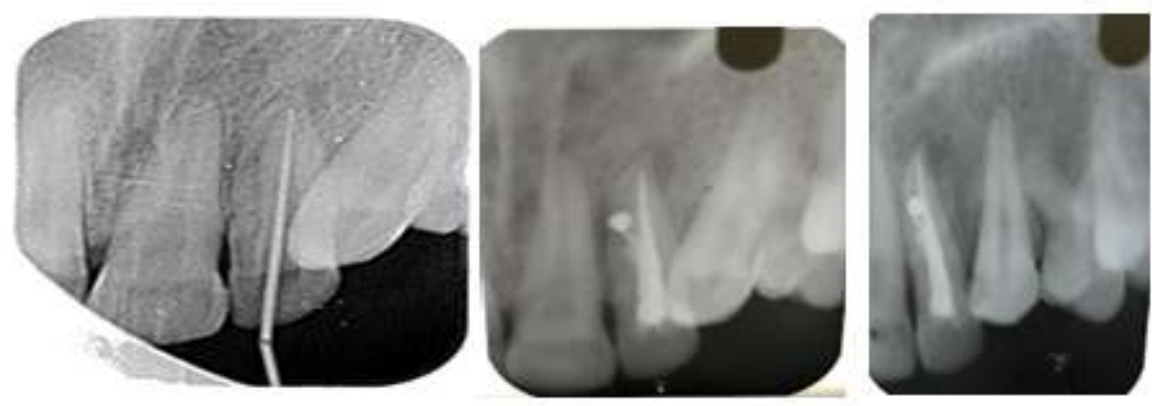

Figure 3:- Mastercone IOPA

Figure 4:- Obturation IOPA

Figure 5:- 12 months follow up IOPA

\section{Discussion}

Maxillary incisors and more specifically the lateral incisors are often affected by abnormalities such as talon cusp, dens invagination, palatogingival groove and $\mathrm{C}$ shaped canals. The irregular areas in C-shaped root canal system often may harbor soft tissue remnant or infected debris and/or bacterial biofilm, leading to inadequate cleaning and root canal obturation. Many modified techniques have been proposed to endodontically manage such cases. The canal spaces should be prepared normally. However, the isthmus should be prepared by circumferential filing with hand files and copious irrigation. Use of ultrasonics is very beneficial in such cases as it helps in complete cleaning of the web and fin areas of the c shaped canal.

Three dimensional obturation of the canal is the key to successful endodontic treatment in such cases.Conventional radiography plays an important role in root canal assessment, however; it provides a twodimensional image of a three-dimensional structure. Use of CBCT and endodontic microscope aids in recognition and decision making in most complicated cases. The existence of $\mathrm{C}$-shaped canal in the invaginated 
teeth has already been reported. It is still a matter of debate whether this type of canal is most routinely seen in invaginated teeth or not.

\section{Conclusion}

When sound principles of biomechanical preparation and obturation are followed, the long-term prognosis for the $\mathrm{C}$-shaped root becomes predictable. The use of the ultrasonics, (MTA Fillapex), thermoplasticizedobturation techniques, SAF system, CBCT and Endodontic Microscopes can be successfully used to treat such cases. MTA based sealers help in proper sealing of the canal and also helps in regenerating bone and inducing cementogenesis.

\section{References}

[1]. Hargreaves K, Cohen S. Pathway of the Pulp. 2011; 10th edition: 180.

[2]. Cleghorn B, Goodacre CH. Ingle's Endodontics. 2008; 6th edition: 162

[3]. Jafarzaheh H, Wu YN. The C-shape root canal configuration: A review. J Endod. 2007; 33 (5):517-23.

[4]. Vikram M. C-shaped canal, an endodontic challenge. Health Renaissance. 2013; 11(1):89-91.

[5]. Ingle JI, Bakland LK, Baumgartner JC (2008) Ingle's Endodontics. (6th edn.), BC Decker Inc., Ontario, USA, pp. 162.

[6]. Maryam Forghani, Conservative Treatment of an Invaginated Maxillary Lateral Incisor with a C-shaped Canal Using Cone-Beam Computed Tomography, IEJ Iranian Endodontic Journal 2015; 10(4): 281-283.

[7]. Ruddle JC. Three-dimensional obturation of the root canal system. Dent Today 1992; 11:28, 30-3, 39.

[8]. Oehlers FA. Dens invaginatus I. Vanations of the invagination process and associated anterior crown forms. Oral Surg Oral Med Oral Pathol. 1957; 10: 1204-18.

[9]. Metzger Z, Teperovich E, Zary R, Cohen R, Hof R. The self-adjusting file (SAF). Part 1: respecting the root canal anatomy- a new concept of endodontic files and its implementation. J Endod 2010; 36:679 90.

[10]. Elumalai D, Kumar A, Tewari RK, Mishra SK, Andrabi SM, Iftekhar H, Alam S. Management of C-shaped root canal configuration with three different obturation systems. Eur J Gen Dent 2015.

[11]. Physical Properties of MTA Fillapex Sealer Rafael PinoVitti, DDS, MSc, PhD, ${ }^{*} \uparrow \mathrm{Carlo}$ Prati, DDS, MSc, $\mathrm{PhD}, \dagger$ EmmanuelJo aoNogueira JOE — Volume 39, Number 7, July 2013

[12]. Rahimi S, Shahi S, Lotfi M, Zand V, Abdolrahimi M, Es'haghi R. Root canal configuration and the prevalence of C-shaped canals in mandibular second molars in an Iranian population. J Oral Sci. 2008; 50(1):9-13.

[13]. Boveda C, Fajardo M. Root canal treatment of an Invaginated maxillary lateral incisor with a C-shaped canal. Quintessence Int. 1999; 30(10):707-11.

[14]. Gound TG. Dens invaginatus--a pathway to pulpal pathology: a literature review. Pract Periodontics Aesthet Dent. 1997; 9(5):58594.

[15]. Maryam Bidar, Conservative Treatment of an Invaginated Maxillary Lateral Incisor with a C-Shaped Canal Using Cone Beam Computed Tomography: A case report J Dent Health Oral DisordTher 2015, 3(1): 00075. 